\title{
The impact of the NHS electronic-alert system on the recognition and management of acute kidney injury in acute medicine
}

\author{
Author: West Midlands Acute Medicine Collaborative ${ }^{\mathrm{A}}$
}

To address inconsistencies in the recognition and management of acute kidney injury (AKI), an electronic-alert (e-alert) system was implemented by NHS England in 2015. This study aimed to describe its impact within acute medicine in the West Midlands. All admissions to included acute medical units were screened for AKI in two phases, before and after the e-alert was introduced. Data describing recognition and management of patients with AKI were collected. In the 10 units that participated in both phases, recognition of AKI by clinicians significantly improved from $67.9 \%$ in 2015 to $76.1 \%$ in 2016 $(p=0.04)$. Further analysis of the data found that the presence of an e-alert had a limited effect on recognition and management, suggesting it was not the primary cause of the improvements. Multiple avenues of research have been recommended to clarify the impact of the e-alert system and to improve deficiencies in management that were identified in the data.

KEYWORDS: Acute kidney injury, definition, recognition, acute medicine, e-alert

\section{Introduction}

Acute kidney injury (AKI) is under-recognised and flaws in management are noted when it is identified. ${ }^{1-3}$ This is a concern as the diagnosis of AKI is associated with a significant mortality; even milder forms are associated with a mortality rate of $7-18 \% .4,5$

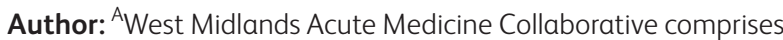
Omar Bani-Saad, Adam Seccombe, Paarul Prinja, Joseph Wheeler, Azeez Olajide, Hadiza Suleiman Gachi, Amie Burbridge, Hesham Kaawan, Mary E Packer, Clare Pollard, Clare Philliskirk, Tyrone Lightbody, Andrea Adjetey, Donna Best, Umar Rahim Bakhsh, Marwa Mattar, Mohammad Saim, Babatunde Adewunmi, Zoe Kimbley, Shelley Raveendran, Ashesh Saha, Paul Francis Spencer Vincent, Charlotte Victoria Reed, Ibrahim Malik, Pradeep Mallisetty, Lois Houlders, Angela Perrett, Alison Pullen, Alison Eastaugh, Alessandra De Serio, Harjinder Kaur Kainth, Craig Pascoe, Saad Arshad Rana, Oloruntoba Fayeye, Llinos Carys Evans, Ranjit Bains, Thomas Holyoake, Leah Jones, Matthew JW Kain, Ibrahim Mustapha, Gregory Packer and Adam Ryder. Author contributions and affiliations can be found in supplementary material S1.
Furthermore, the prevalence of AKI has been reported to be as high as $18 \%$ in acute hospital admissions. . $^{4,6}$

Consensus statements $s^{3,7}$ and guidelines ${ }^{8,9}$ recommend urinalysis, renal ultrasound, drug dose adjustments and avoidance of nephrotoxic medications during the early management of AKI. Intravenous fluid should be considered for all cases. However, if not required, aggressive fluid therapy is harmful ${ }^{10}$ so a preceding fluid assessment is essential. Studies exploring the impact of such management steps have shown a reduction in the incidence and severity of AKI. $3,11,12$

To improve the management of patients with AKI, electronicalert (or 'e-alert') systems have been recommended to support recognition. The benefit of such systems remains unclear. Some studies demonstrated success in changing clinician behaviour and patient outcome. ${ }^{13-16}$ Others failed to do so. ${ }^{17}$ A systematic review of six studies found AKI e-alert systems did not improve survival or reduce renal replacement therapy utilisation. ${ }^{18}$

Despite this uncertainty, an e-alert system for AKI was implemented throughout the NHS in England. ${ }^{19}$ An automated screening process reports AKI and its stage alongside the creatinine result. It is driven by an algorithm based on NICE clinical guideline CG169. ${ }^{8}$ This guidance aimed to improve the recognition and management of AKI, particularly in the initial stages, and focused on generalist specialties such as acute medicine to maximise their impact. The guidelines used the Kidney Disease: Improving Global Outcomes (KDIGO) classification ${ }^{7}$ to identify AKI based on increases in creatinine or reductions in urine output. The target date for nationwide implementation of the e-alert was 9 March 2015.

The aim of this study was to describe the recognition and management of AKI in the West Midlands before and after the implementation of the NHS AKI e-alert system. A secondary aim was to describe how severity (defined by KDIGO stage) was associated with recognition and management.

\section{Method}

This observational study was reported according to the 'Strengthening the Reporting of Observational Studies in Epidemiology' (STROBE) statement. ${ }^{20}$ A protocol was designed in advance and agreed by all members of the West Midlands Acute Medicine Collaborative (WAM-C): a trainee-led research collaborative. $^{21}$ 
The study was registered as an audit at each site in the West Midlands. Data were collected prospectively in two phases, before and after implementation of the e-alert system, on six separate days as determined by local site team at each site. The on-call medical team were unaware of the study prior to completion.

Phase one took place between 2 February and 16 March 2015 at 14 hospital sites. All data were collected before the e-alert system was active at each site. Phase two took place at 11 hospital sites between 1 April and 22 May 2016, 1 year following implementation of the e-alert system. Ten sites were involved in both phases of data collection.

All patients admitted onto the acute medical unit (AMU) during each collection day were manually screened for AKI. Medical patients admitted directly to other wards, including intensive care, were not included in the study. Numbers of patients without AKI were recorded in phase one to calculate prevalence. AKI was defined according to the KDIGO definition. ${ }^{7}$ As per 2011 Renal Association guidelines, ${ }^{9}$ and recent articles, ${ }^{22-25}$ baseline creatinine was defined as the lowest creatinine level recorded within 3 months. If a 3-month creatinine was not available, then AKI was not diagnosed, assuming other criteria were not met. This was with the aim of maximising specificity, ie ensuring all identified cases had AKI rather than chronic kidney disease (CKD): defined by KDIGO as a decline in kidney function for $>3$ months. ${ }^{26}$

Recognition of AKI by clinicians was determined by the presence of a documented diagnosis of AKI in the medical records. Clinical recognition or recognition by the e-alert system was calculated as a percentage of AKI identified by the study team. The successful completion of four management strategies within the first 24 hours (see Table 1) was also based on medical records alone. If a variable was not recorded, it was assumed the standard had not been met. The stage of each AKI was recorded in phase two only.

Data were recorded on a standardised Microsoft Excel 2010 spreadsheet by the local site team, anonymised and then returned

\section{Table 1. Study criteria}

\begin{tabular}{ll}
$\begin{array}{l}\text { Standard } \\
\begin{array}{l}\text { 1. Recognition } \\
\text { of AKI }\end{array}\end{array}$ & $\begin{array}{l}\text { If a diagnosis of AKI is documented in the } \\
\text { initial clerking or senior review. }\end{array}$ \\
$\begin{array}{ll}\text { 2. Nephrotoxic } \\
\text { medication } \\
\text { stopped }\end{array}$ & $\begin{array}{l}\text { If a nephrotoxic medication that was } \\
\text { prescribed before the AKI diagnosis had } \\
\text { been stopped. Answer 'N/A' if the patient is } \\
\text { not on a nephrotoxic medication. }\end{array}$ \\
$\begin{array}{ll}\text { 3. Urinalysis } \\
\text { performed }\end{array}$ & $\begin{array}{l}\text { If a urinalysis result was documented in the } \\
\text { medical records. Answer 'N/A' if the patient } \\
\text { is anuric or awaiting an intervention to drain }\end{array}$ \\
$\begin{array}{ll}\text { 4. Renal } \\
\text { ultrasound } \\
\text { requested if } \\
\text { appropriate }\end{array}$ & $\begin{array}{l}\text { Answer 'N/A' unless the cause of AKI is } \\
\text { unknown or is likely to be obstruction based } \\
\text { on medical records. Standard met if a }\end{array}$ \\
$\begin{array}{l}\text { 5. Volume } \\
\text { assessment }\end{array}$ & $\begin{array}{l}\text { If any documentation of volume status is } \\
\text { found in the medical records (including terms } \\
\text { such as dry, overloaded, dehydrated). }\end{array}$ \\
\hline $\begin{array}{l}\text { This is a list of the audit standards used to assess the recognition and } \\
\text { management of AKI as described in the study protocol. AKI =acute kidney } \\
\text { injury. }\end{array}$
\end{tabular}

Table 2. Characteristics of AKI cases in phase one

\section{and phase two}

\begin{tabular}{|c|c|c|c|}
\hline \multicolumn{2}{|c|}{ Variable studied } & $\begin{array}{l}\text { Phase } \\
\text { one }\end{array}$ & $\begin{array}{l}\text { Phase } \\
\text { two }\end{array}$ \\
\hline \multicolumn{2}{|c|}{ Mean age, years (SD) } & $\begin{array}{l}75.6(60.9- \\
90.3)\end{array}$ & $\begin{array}{l}75.9(61.1- \\
90.6)\end{array}$ \\
\hline Gender, n (\%) & Female & $184(52.2)$ & $136(52.1)$ \\
\hline \multirow{3}{*}{$\begin{array}{l}\text { Criteria used } \\
\text { to diagnose } \\
\text { AKI, n ( \%) }\end{array}$} & $\begin{array}{l}\text { Creatinine rise } \geq 50 \% \\
\text { increase from baseline }\end{array}$ & $293(83)$ & $219(84)$ \\
\hline & $\begin{array}{l}\text { Creatinine rise } \geq 26 \\
\mu \mathrm{mol} / \mathrm{l} \text { in } 48 \mathrm{hr}\end{array}$ & $57(16)$ & $41(16)$ \\
\hline & $\begin{array}{l}\text { Urine output }<0.5 \mathrm{~mL} / \\
\mathrm{kg} / \mathrm{hr} \text { over } 6 \text { hours }\end{array}$ & $2(<1)$ & $0(0)$ \\
\hline
\end{tabular}

This table compares the age, gender and chosen diagnostic criteria for AKI between phase one and phase two. AKI = acute kidney injury; SD = standard deviation.

to the study lead for analysis. Apparent differences were tested using $\chi^{2}$ test where appropriate.

\section{Results}

2,791 patients were screened in 14 AMUs across the West Midlands during phase one. 354 AKI cases were identified, giving an estimated prevalence of AKI in acute medical admissions of $12.7 \%$ (95\%, confidence interval $=11.5-14.0)$. In phase two, 265 AKI cases were identified across 11 AMUs. A total of six patients were approaching the end of life and so were excluded from analysis: two in phase one, four in phase two. Age, gender and criteria used to diagnosed AKI were comparable between the two phases (see Table 2).

As summarised in Table 3, 73.4\% of AKI cases across both phases had a documented diagnosis, the proxy for clinical recognition. Urinalysis and volume assessment were performed in $50.2 \%$ and $54.3 \%$ of AKI cases, respectively. When a patient was taking a nephrotoxic medication, it was stopped in $81.5 \%$ of cases and, when an ultrasound was deemed necessary by the study team, it was requested in $64.4 \%$ of cases.

Table 3. Overall recognition and management of

AKI by clinicians

\section{Total Documented Not} documented

Number (\%) $\quad 613(100) \quad 450(73.4) \quad 163(26.5)$

Volume assessment, $333(54.3) \quad 271(60.2) \quad 62(38.0)$

$\mathrm{n}(\%)$

Urinalysis, $\mathrm{n}(\%) \quad 302(50.2) \quad 242(54.3) \quad 60(38.5)$

Nephrotoxics $\quad 326(81.5) \quad 272(88.0) \quad 54(59.3)$

stopped, n (\%)

Ultrasound $\quad 94(64.4) \quad 87(74.4) \quad 7(24.1)$

requested, $\mathrm{n}(\%)$

This table illustrates the number and percentage of AKI cases that were documented and appropriately managed using combined data from phase one and phase two. AKI = acute kidney injury. 
Table 4. Recognition and management of acute kidney injury by severity

\begin{tabular}{|c|c|c|c|}
\hline Stage of AKI & Stage 1 & Stage 2 & Stage 3 \\
\hline Number (\%) & $147(62.8)$ & $63(26.9)$ & $51(21.8)$ \\
\hline Documented, n (\%) & $92(62.5)$ & $58(92.1)$ & $46(90.2)$ \\
\hline E-alert present, n (\%) & $96(65.3)$ & $53(84.1)$ & $45(88.2)$ \\
\hline Volume assessment, n (\%) & $79(53.7)$ & $39(61.9)$ & $37(72.5)$ \\
\hline Urinalysis, n (\%) & $74(52.5)$ & $34(54.8)$ & $35(71.4)$ \\
\hline $\begin{array}{l}\text { Nephrotoxic medications } \\
\text { stopped, n (\%) }\end{array}$ & $71(77.1)$ & $36(85.7)$ & $33(94.3)$ \\
\hline Ultrasound requested, n (\%) & $10(38.5)$ & $12(92.3)$ & $17(85.0)$ \\
\hline
\end{tabular}

There was a significant improvement in all management standards if AKI was documented by the clinical team. Three of the standards showed a $50 \%$ increase: volume assessment $\left(\chi^{2}=23.74 ; p=<0.0001\right)$, urinalysis $\left(\chi^{2}=11.54 ; p=0.0006\right)$, and nephrotoxics stopped $\left(\chi^{2}=38.36 ; p=<0.0001\right)$. A renal ultrasound request, when appropriate, was three times more likely with a documented diagnosis $\left(\chi^{2}=25.56 ; p=<0.0001\right)$.

During phase two, the stage of AKI was recorded. $55.1 \%, 24.6 \%$, and $20.3 \%$ were noted to have stage 1,2 and 3 respectively (see Table 4). Stage 1 AKI was the least likely to be documented. Stage 2 and 3 were both documented in approximately $90 \%$ of cases. These differences in documentation were significant $\left(\chi^{2}=28.22\right.$; $p=<0.0001)$. The stage of AKI had an impact on management standards that differed from its impact on documentation. With the exception of ultrasound requesting, all management standards were more likely to be performed for AKI stage 3 than AKI stage 2. However, none of these differences was significant in isolation.
Table 5. Changes in recognition and management

between phase one and phase two

\begin{tabular}{|c|c|c|}
\hline & Phase one & Phase two \\
\hline Number of AKI cases & 262 & 234 \\
\hline Documented, n (\%) & $178(67.9)$ & $178(76.1)$ \\
\hline Volume assessment, $\mathrm{n}(\%)$ & $129(49.2)$ & $137(58.5)$ \\
\hline Urinalysis, n (\%) & $109(41.8)$ & $124(54.1)$ \\
\hline $\begin{array}{l}\text { Nephrotoxic medications stopped, } \\
\text { n (\%) }\end{array}$ & $143(80.8)$ & $128(83.1)$ \\
\hline Ultrasound requested, n (\%) & $47(65.3)$ & $35(63.6)$ \\
\hline
\end{tabular}

Comparisons between phase one and two were restricted to the 10 hospitals that participated in both phases to better match patient populations and working practices at the same sites. This decision was made following data collection. As shown in Table 5, the proportion of documented AKI cases significantly increased between phase one and phase two $\left(\chi^{2}=4.03 ; p=0.04\right)$. This increase was also seen for volume assessment $\left(\chi^{2}=4.31 ; p=0.04\right)$ and urinalysis $\left(\chi^{2}=7.50 ; p=0.006\right)$.

To analyse the impact of the e-alert system on these changes, AKI cases with and without an e-alert, and with and without a documented diagnosis were compared in phase two (see Table 6). The proportion of AKI identified by the e-alert system (74.3\%) was similar to the proportion documented by clinicians $(74.7 \%)$. However, recognition was discordant between clinicians and the e-alert system in one in three cases of AKI. Of note, the presence of an e-alert was not associated with a significant improvement in documentation or management standards, with the exception of ultrasound requesting $\left(\chi^{2}=7.56 ; p=0.006\right)$. This was in contrast

Table 6 . The variation in recognition and management depending on the presence of a documented diagnosis and an e-alert

\begin{tabular}{|c|c|c|c|c|c|c|c|}
\hline & Phase two total & e-alert & No e-alert & Documented & Undocumented & Both & Neither \\
\hline $\begin{array}{l}\text { Number of AKI cases (\% of } \\
\text { total AKI cases) }\end{array}$ & 234 & $179(76.5)$ & $55(23.5)$ & $178(76.1)$ & $56(23.9)$ & $138(59)$ & $15(6.4)$ \\
\hline $\begin{array}{l}\text { Documented, } n \text { (\% of total } \\
\text { AKI cases) }\end{array}$ & $178(76.1)$ & $138(77.1)$ & $40(72.7)$ & $178(100)$ & $0(0)$ & $138(100)$ & $0(0)$ \\
\hline $\begin{array}{l}\text { Volume assessment, } \mathrm{n} \text { (\% of } \\
\text { total AKI cases) }\end{array}$ & $137(58.5)$ & $105(58.7)$ & $32(58.2)$ & $117(65.7)$ & $20(35.7)$ & $91(65.9)$ & $6(40)$ \\
\hline Urinalysis, $\mathbf{n}$ (\% unless anuric) & $124(54.1)$ & $98(56.3)$ & $26(47.3)$ & $99(56.6)$ & $25(46.3)$ & $76(56.2)$ & $3(20)$ \\
\hline $\begin{array}{l}\text { Nephrotoxic medications } \\
\text { stopped, } \mathrm{n} \text { (\% of total patients } \\
\text { on nephrotoxic medication) }\end{array}$ & $128(83.1)$ & $97(82.9)$ & $31(83.8)$ & $109(90.1)$ & $19(57.6)$ & $85(90.4)$ & $7(70)$ \\
\hline $\begin{array}{l}\text { Ultrasound requested, } \mathrm{n} \text { (\% } \\
\text { when ultrasound is indicated) }\end{array}$ & $35(63.6)$ & $30(73.2)$ & $5(35.7)$ & $34(70.8)$ & $1(14.3)$ & $29(78.4)$ & $0(0)$ \\
\hline
\end{tabular}


to the presence of a documented diagnosis, which continued to be associated with a significant increase in all four management standards in this dataset, apart from urinalysis. The presence of both an e-alert and a documented diagnosis was not associated with a significant change to any of the four management standards compared with a documented diagnosis alone.

\section{Discussion}

This multicentre study explored the recognition and management of AKI by clinicians in AMUs across the West Midlands and how these standards changed following the implementation of the NHS AKI e-alert system in 2015. While there was a significant improvement in the recognition and management of AKI between phase one and phase two, further analysis of the data suggested this was not due to the e-alert system.

AKI was frequently missed by clinicians; a documented diagnosis was absent in one in every four cases. Analysis of AKI management found similar deficiencies. Both urinalysis and volume assessment were absent from one in two medical records, one in three cases did not have an ultrasound request when appropriate, and nephrotoxic medications were not stopped for one in five patients when relevant.

In addition, the data support the view that recognition by itself is not the answer. Even for documented diagnoses, deficiencies were noted. This was particularly true for volume assessment, completed in $60.2 \%$ of documented AKI cases, and urinalysis, completed in $54.3 \%$ of documented cases. Volume assessment is acknowledged to be challenging, ${ }^{27}$ so any comment and examination linked to volume or fluid balance were accepted, setting a low bar. The failure to meet this standard is a concern given the harm that unnecessary intravenous fluid can cause. ${ }^{10}$ Moreover, this study only identified community-acquired AKI. Because the quality of care is worse for patients who develop AKI after admission (ie hospital-acquired AKI), ${ }^{2}$ the above failures in recognition and management could be an overestimate.

Recognition and management varied with stage of AKI. Nearly one in three cases of stage 1 AKI had no documented diagnosis. In contrast, only one in ten cases of stage 2 and 3 AKI were not documented. Management was also better for more severe stages of AKI. Of note, the management of stage 3 AKI appeared to be better than that of stage 2 . However, these differences were not significant. The lower documentation rates for stage 1 AKI could reflect the failure of clinicians to recognise the condition. Alternatively, clinicians may fail to document a diagnosis because they underestimated its significance. This would explain why a proportion of patients with undocumented AKI were still appropriately managed and might explain the possibility that stage 3 AKI is managed better than stage 2 despite identical documentation rates. The breakdown of AKI by stage was similar to the breakdown of AKI severity found in other studies. ${ }^{28}$

Between phase one and phase two, there was a significant improvement in documentation and in two of the four management standards (volume assessment and urinalysis). However, while a documented diagnosis was associated with improvements in three management steps, an e-alert was only associated with improvements in ultrasound requesting. Furthermore, the documented diagnosis and e-alert disagreed in one in three cases. These findings suggest improvements in management were not linked to the e-alert, and that the e-alert system is having a limited impact upon clinical decision making.
Alert fatigue is a well-recognised phenomenon which offers a possible explanation. An interventional study of an AKI-alert system noted $78.1 \%$ of interruptive alerts were deferred by clinicians. ${ }^{29}$ Because the NHS e-alert is not interruptive, its impact is likely to be lower and a higher proportion of e-alerts might be ignored. The many other campaigns, quality improvement projects and care bundles that have been completed since the AKI-themed National Confidential Enquiry into Patient Outcome and Death (NCEPOD) report in $2009^{2}$ and NICE AKI guidelines in $2013^{8}$ may all have contributed to the improvements seen between phase one and phase two.

By excluding patients who had no creatinine result within the last 3 months, some AKI cases will have been excluded from this study. This offers an explanation for the AKI prevalence of $12.7 \%$, lower than the $17.7 \%$ found in a similar UK study performed in AMUs. ${ }^{30}$ The choice of baseline did maximise the specificity of the AKI definition, however, and limited the inclusion of patients with a progression of CKD. As a result, all identified AKI cases should have been recognised as AKI by clinicians and the e-alert system and managed appropriately.

The e-alert's algorithm (based on that described in the NICE guidelines) ${ }^{8}$ defines the baseline creatinine using the lowest creatinine result in the last 7 days or the median of all results within the last year. When creatinine rises occur in the preceding 12 months, this approach can miss AKI, which may explain the high proportion of stage 1 AKI that was missed by the e-alert system. Currently, there is a limited evidence base to support any definition of the baseline creatinine, which is acknowledged in the literature $^{22,31}$ and by KDIGO. ${ }^{\text {? }}$

A further limitation of this study was the decision to judge the standards solely based on documentation. The recognition of AKI by clinicians was assumed to be equivalent to a documented diagnosis. However, it is likely that documentation was forgotten on occasion despite the clinician being aware of the AKI.

This study highlights multiple avenues for research. Exploring the decision-making process that leads to a clinical diagnosis of AKI could provide multiple benefits; identifying knowledge gaps for all grades of clinician, understanding the interaction between clinicians and the e-alert algorithm, and defining how experienced clinicians determine the baseline creatinine, perhaps offering a new approach for further study. Subsequent research to support a single definition of the baseline creatinine would provide an evidence base to support future AKI guidelines. Addressing this issue is particularly important as even a small increase in creatinine causes mortality to rise. ${ }^{32}$

Finally, future studies that explore why each management standard was not completed would allow improvement measures to be appropriately designed and targeted. For example, a number of issues could prevent a urinalysis from being performed, including patient incontinence, a lack of equipment or staff, or a communication failure.

\section{Conclusion}

The AKI e-alert system and the algorithm that drives it remains a cornerstone of NHS policy. However, evidence to support its impact is lacking. While this study did note an improvement in AKI recognition and management between phase one and phase two, this change did not seem to be linked to the e-alert system. Furthermore, the documentation of AKI by clinicians did not appear to be linked to the presence of an e-alert. Several avenues 
of research have been identified that should be explored before further attempts to improve the recognition and management of AKI are made. Addressing these research aims should be a priority given the ongoing deficiencies in the recognition and management of AKI identified in this study.

\section{Supplementary material}

Additional supplementary material may be found in the online version of this article at www.clinmed.rcpjournal.org:

S1 - Authorship.

\section{References}

1 Stevens PE, Tamimi NA, Al-Hasani MK et al. Non-specialist management of acute renal failure. QJM 2001;94:533-40.

2 National Confidential Enquiry into Patient Outcome and Death. Adding insult to injury: A review of the care of patients who died in hospital with a primary diagnosis of acute kidney injury (acute renal failure). London: NCEPOD, 2009.

3 Mehta RL, McDonald B, Gabbai F et al. Nephrology consultation in acute renal failure: does timing matter? Am J Med 2002;113:45661.

4 Uchino S, Bellomo R, Goldsmith D, Bates S, Ronco C. An assessment of the RIFLE criteria for acute renal failure in hospitalized patients. Crit Care Med 2006:34:1913-7.

5 Chertow GM, Burdick E, Honour M, Bonventre JV, Bates DW. Acute kidney injury, mortality, length of stay, and costs in hospitalized patients. J Am Soc Nephrol 2005;16:3365-70.

6 Lafrance JP, Miller DR. Acute kidney injury associates with increased long-term mortality. J Am Soc Nephrol 2010;21:345-52.

7 Kellum J, Lameire N, Aspelin P et al. Kidney Disease: Improving Global Outcomes (KDIGO) Acute Kidney Injury Work Group. KDIGO clinical practice guideline for acute kidney injury. Kidney Int Suppl 2012:2:1-138.

8 National Institute for Health and Care Excellence. Acute kidney injury: prevention, detection and management. Clinical guideline [CG169]. London: NICE, 2013.

9 Lewington A, Kanagasundaram S. Clinical Practice Guidelines: Acute Kidney Injury, 5th ed. Renal Association, 2011.

10 Himmelfarb J, Joannidis M, Molitoris B et al. Evaluation and initial management of acute kidney injury. Clin J Am Soc Nephrol 2008:3:962-7.

11 Perez-Valdivieso JR, Bes-Rastrollo M, Monedero P, de Irala J, Lavilla FJ. Prognosis and serum creatinine levels in acute renal failure at the time of nephrology consultation: an observational cohort study. BMC Nephrol 2007:8:14.

12 Balasubramanian G, Al-Aly Z, Moiz A et al. Early nephrologist involvement in hospital-acquired acute kidney injury: a pilot study. Am J Kidney Dis 2011:57:228-34.

13 Kolhe NV, Staples D, Reilly T et al. Impact of compliance with a care bundle on acute kidney injury outcomes: A prospective observational study. PLoS One 2015;10:e0132279.

14 Colpaert K, Hoste EA, Steurbaut K et al. Impact of real-time electronic alerting of acute kidney injury on therapeutic intervention and progression of RIFLE class. Crit Care Med 2012;40:1164-70.

15 Rind DM, Safran C, Phillips RS et al. Effect of computer-based alerts on the treatment and outcomes of hospitalized patients. Arch Intern Med 1994;154:1511-7.
16 Cho A, Lee JE, Yoon JY et al. Effect of an electronic alert on risk of contrast-induced acute kidney injury in hospitalized patients undergoing computed tomography. Am J Kidney Dis 2012;60:74-81.

17 Wilson FP, Shashaty M, Testani ] et al. Automated, electronic alerts for acute kidney injury: a single-blind, parallel-group, randomised controlled trial. Lancet 2015;385:1966-74.

18 Lachance P, Villeneuve PM, Rewa OG et al. Association between e-alert implementation for detection of acute kidney injury and outcomes: a systematic review. Nephrol Dial Transplant 2017;32:265-72.

19 NHS England. Acute Kidney Injury Warning Algorithm Best Practice Guidance. London: NHS England, 2014.

20 von Elm E, Altman DG, Egger $\mathrm{M}$ et al. The Strengthening the Reporting of Observational Studies in Epidemiology (STROBE) statement: guidelines for reporting observational studies. Lancet 2007;370:1453-7.

21 West Midlands Acute Medicine Collaborative. www.wmacutemed.co.uk.

22 Anathhanam S, Lewington AJ. Acute kidney injury. J R Coll Physicians Edinb 2013;43:323-8.

23 Mohamed F, Endre Z, Jayamanne S et al. Mechanisms underlying early rapid increases in creatinine in paraquat poisoning. PLoS One 2015:10:e0122357.

24 Rodriguez E, Arias-Cabrales C, Bermejo S et al. Impact of recurrent acute kidney injury on patient outcomes. Kidney Blood Press Res 2018:43:34-44

25 Cordova-Sanchez BM, Herrera-Gomez A, Namendys-Silva SA. Acute kidney injury classified by serum creatinine and urine output in critically ill cancer patients. Biomed Res Int 2016;2016:6805169.

26 Kidney Disease: Improving Global Outcomes (KDIGO) Acute Kidney Injury Work Group. KDIGO clinical practice guideline for acute kidney injury: Online Appendix B. KDIGO, 2012: https://kdigo.org/wp-content/ uploads/2016/10/KDIGO-AKI-Suppl-Appendices-A-F_March2012.pdf.

27 Seccombe A, Sapey E. What is the evidence base for fluid resuscitation in acute medicine? Clin Med 2018;18:225-30.

28 Wonnacott A, Meran S, Amphlett B, Talabani B, Phillips A. Epidemiology and outcomes in community-acquired versus hospital-acquired AKI. Clin J Am Soc Nephrol 2014;9:1007-14.

29 McCoy AB, Waitman LR, Gadd CS et al. A computerized provider order entry intervention for medication safety during acute kidney injury: a quality improvement report. Am J Kidney Dis 2010;56:832-41.

30 Finlay S, Bray B, Lewington AJ et al. Identification of risk factors associated with acute kidney injury in patients admitted to acute medical units. Clin Med 2013;13:233-8

31 Gaiao S, Cruz DN. Baseline creatinine to define acute kidney injury: is there any consensus? Nephrol Dial Transplant 2010;25:3812-4.

32 Mangano CM, Diamondstone LS, Ramsay JG et al. Renal dysfunction after myocardial revascularization: risk factors, adverse outcomes, and hospital resource utilization. The multicentre study of Perioperative Ischemia Research Group. Ann Intern Med 1998;128:194-203.

Address for correspondence: Dr Adam Seccombe, acute medicine research fellow and honorary acute medicine consultant, Acute Medical Unit, University Hospital Birmingham NHS Foundation Trust, Birmingham B15 2TH, UK. Email: a.seccombe@nhs.net 